\title{
Peptococcaceae, a New Family To Include the Gram-Positive, Anaerobic Cocci of the Genera Peptococcus, Peptostreptococcus, and Ruminococcus
}

\author{
MORRISON ROGOSA \\ Microbial-Physiology Section, Laboratory of Microbiology and Immunology, \\ National Institute of Dental Research, Bethesda, Maryland 20014
}

\begin{abstract}
Peptococcaceae is proposed as a new family in the order Eubacteriales to include three genera of presently known gram-positive, anaerobic, coccal organisms: Peptococcus Kluyver and van Niel, Peptostreptococcus Kluyver and van Niel, and Ruminococcus Sijpestein. The type genus of this family is Peptococcus. Descriptions of these taxa are given, and where necessary the original descriptions have been expanded and brought up to date.
\end{abstract}

The genera Peptococcus and Peptostreptococcus were named by Kluyver and van Niel (1), who assigned them to the tribes Micrococceae and Streptococceae, respectively, within the family Micrococcaceae. Peptococcus was the tenth listed genus in Micrococceae and was described as follows.

"10. Peptococcus nov. gen. ${ }^{2}$ )

Spherical cells, either immotile or motile. No endospores formed. Chemoheterotrophic, anaerobic, capable of fermenting protein decomposition products. Gram positive.

The type species is Peptococcus niger (Hall).

${ }^{2}$ The various anaerobic, Gram positive cocci placed by Prévot (1.c.) in the genera Diplococcus, Gaffkya, Staphylococcus and Micrococcus might well be united in this genus, as long as further particulars regarding their katabolism are lacking."

Peptostreptococcus was the first listed genus in Streptococceae and was defined as follows (1).

"1. Peptostreptococcus gen. nov.

Spherical cells, generally occurring in chains. Immotile. No endospores formed. Chemoheterotrophic, anaerobic, capable of fermenting protein decomposition products with the formation of carbon dioxide, hydrogen, and other unknown products. Gram positive.

The type species is Peptostreptococcus anaerobius (Kronig et Menge)."

From the foregoing, it is clear that Peptostreptococcus was differentiated from Peptococcus mainly by the ability of Peptostreptococcus to form chains; both genera were "capable of fermenting protein decomposition products." In the case of Peptostreptococcus, "carbon dioxide, hydrogen, and other unknown products" accumulated in cultures. Adequate analytical methods for the determination of lactic acid were then available, and it is significant that there was no mention of lactic acid as a product with either genus (1). Clearly, lactic acid could have been nothing more than a very minor catabolic product, at best.

Streptococcus was the second genus listed in the tribe Streptococceae (1); however, in emphatic contrast to Peptostreptococcus, the genus Streptococcus fermented carbohydrates "with the practically exclusive formation of lactic acid." As is pointed uut later in this paper, Peptostreptococcus and Streptococcus have often been confused, simply because both genera consist of chain-forming spherical organisms. There is little or no doubt that investigators generally ignored the great catabolic differences between these genera ( 2 ; Bergey's Manual of Determinative Bacteriology, 7th ed.).

In addition to Peptococcus and Peptostreptococcus, the genus Ruminococcus Sijpestein has never been unequivocally allocated to any suprageneric taxon. Sijpestein (Ph.D. Thesis, University of Leiden, the Netherlands, 1948) diffidently suggested that Ruminococcus be included with Streptococcus in Streptococceae; however, she and subsequent investigators clearly recognized that the organisms comprising Ruminococcus are not lactic acid bacteria because lactate is not a major product, but, if present, is rather generally a very minor or trace 
constituent of the fermentation products. This, plus the fact that strains of Ruminococcus, have been variously described as gram-positive, gram-variable, or gram-negative, tended to obfuscate the relationship of these cellulolytic cocci to organisms in other genera and suprageneric taxa. Recently, however, electron microscopic examination of thin sections of cells from authentic cultures of Ruminococcus flavefaciens, the type species, and from $R$. albus, the other known species, revealed that cell walls of all examined cultures have the generally characteristic dense, not triple-tracked anatomy associated with gram-positive cells (Roger M. Cole, personal communication; details to be published subsequently). Therefore, whatever the reasons for variations in Gram stain results in the hands of different investigators, Ruminococcus is here considered to be gram-positive.

The author recently proposed (3) the family name Veillonellaceae to accommodate the known gram-negative, anaerobic cocci comprising the genera Veillonella Prévot, Acidaminococcus Rogosa, and Megasphaera Rogosa.

Similarly, the family name Peptococcaceae is here proposed for the gram-positive, anaerobic cocci of the genera Peptococcus Kluyver and van Niel, Peptostreptococcus Kluyver and van Niel, and Ruminococcus Sijpestein. The type genus of this family is Peptococcus. Updated descriptions of these taxa follow.

Peptococcaceae fam. nov.

Pep.to.coc.ca'ce.ae. M. L. mas.n. Peptococcus type genus of the family; -aceae ending to denote a family; M. L. fem. pl. n. Peptococcaceae the Peptococcus family.

Cocci varying in diameter $(0.5$ to $2.5 \mu \mathrm{m})$. Occur singly, in pairs, tetrads, and irregular masses, occasionally in three-dimensional cubic packets. Some organisms may form short or long chains with some elongation of individual elements. Flagella, motility, and endospores are absent. Gram-positive but may stain equivocally. Anaerobic. Chemoorganotrophic with complex nutritional requirements. Gas, consisting of $\mathrm{CO}_{2}$ and usually $\mathrm{H}_{2}$, is produced in cultures from amino acids, or carbohydrates, or both; $\mathrm{H}_{2} \mathrm{~S}$ may also be detectable. Carbohydrates may or may not be fermented. In complex media the major nongaseous products are lower fatty acids; in some cases, succinate or ethanol may also be major products. Lactate, if produced, is generally not a major product.

The genera of Peptococcaceae are (i) Peptococcus Kluyver and van Niel, 1936; type species: $P$. niger (Hall) Kluyver and van Niel; (ii) Peptostreptococcus Kluyver and van Niel, 1936; type species: P. anaerobius (Krönig)
Kluyver and van Niel; (iii) Ruminococcus Sijpestein, 1948; type species: $R$. flavefaciens Sijpestein.

According to the classification of the bacteria in Bergey's Manual, 7th ed., the family Peptococcaceae is placed in the order Eubacteriales.

The descriptions of Peptococcus, Peptostreptococcus, and Ruminococcus are up-dated as follows (for each taxon, the characters in italics are the major defining ones and are possessed by all members of the taxon).

Peptococcus Kluyver and van Niel, 1936.

Spheres, usually 0.5 to $1.0 \mu \mathrm{m}$, exceptionally up to $1.6 \mu \mathrm{m}$, in diameter. Occur singly, in pairs, tetrads, or irregular masses but not in three-dimensional cubical packets. Long chains are not formed, and short chains are infrequent. Flagella, motility, and spores are absent. Grampositive. Anaerobic. Weak catalase activity may be present. Chemoorganotrophic with complex nutritional requirements. Peptones, amino acids, and purines or pyrimidines can be used as major energy sources. In complex media, various combinations of the $C_{1}$ to $C_{4}$ lower volatile fatty acids, $\mathrm{CO}_{2}, \mathrm{H}_{2}$, and ammonia tend to be major products from amino acids; some lactate is also produced from histidine, but lactate is not a major product in glucose complex media. Lactate and malate are not fermented. A number of organisms produce gas from pyruvate but generally not from citrate or tartrate. Saccharoclastic activity is often limited or absent, and added carbohydrates do not appear to be essential for growth (although growth may occasionally be enhanced) in complex media. With some organisms, the addition of fatty acid compounds (such as oleate or Tween 80 ) to complex media may stimulate growth or carbohydrate fermentation.

Isolated from the human female urogential tract, the human intestinal and respiratory tracts, inflamed appendices, a case of cystitis, pleurisy, the gums, postpartum septicemia, tonsils, plasma, skin, tidal bay mud, and caseous lymphadenitis in sheep. Sensitive to penicillin $G$ (1 to 10 units $/ \mathrm{ml}$, and often less than 1 unit $/ \mathrm{ml}$ ). Pathogenicity is probable but at present not clear because isolations are very frequently from sites where other potential pathogens are present. Niel.

Type species: $P$. niger (Hall) Kluyver and van

Peptostreptococcus Kluyver and van Niel, 1936.

Spherical to ovoid cells sometimes lengthened to appear pointed; diameter, 0.7 to 1.0 $\mu \mathrm{m}$, occasionally somewhat less. Occur in pairs 


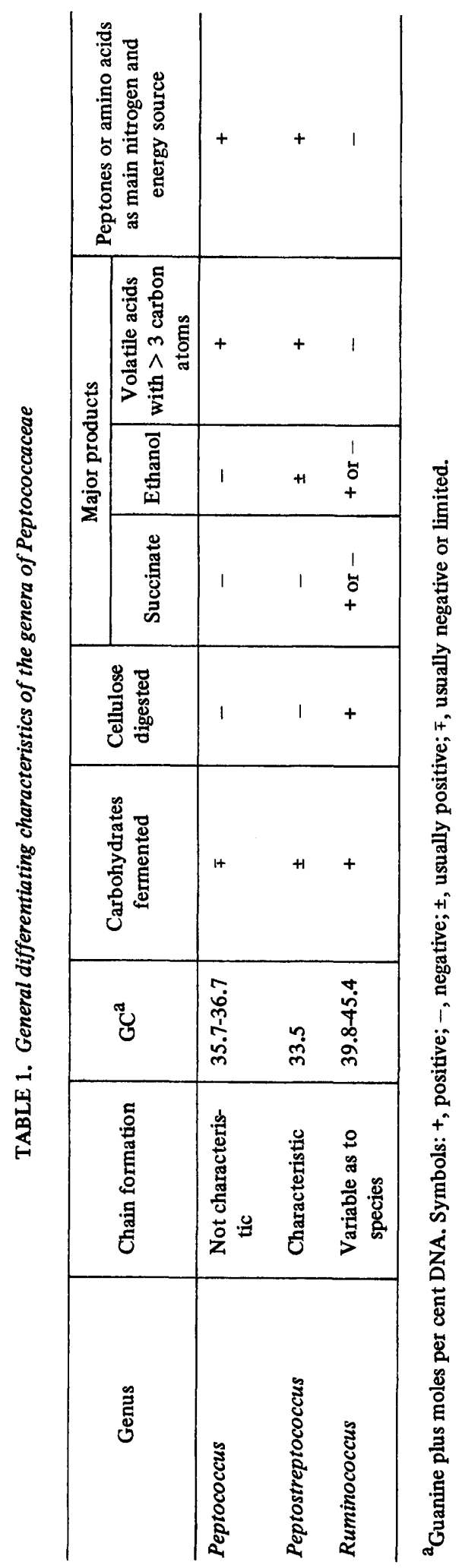

and in short or long chains. No flagella. No motility. No spores. Gram-positive. Anaerobic. Complex media for growth. Where tested, strains have been sensitive to penicillin G (10 units $/ \mathrm{ml}$, and often less than 1 unit $/ \mathrm{ml})$. Catalase negative. Nitrates not reduced. Indole generally not produced. Gelatin generally not liquefied, and coagulated proteins not attacked. Very rarely hemolytic. In complex media, a fetid odor and gas (mainly $\mathrm{CO}_{2}$, with some $\mathrm{H}_{2}$ and $\mathrm{H}_{2} \mathrm{~S}$ ) and large quantities of $\mathrm{C}_{1}$ to $\mathrm{C}_{6}$ volatile acids are produced; lactate is either not produced or is present in at best trace amounts. Ammonia is produced, but alkaline reaction may be prevented by acid produced in carbohydrate media; acetylmethylcarbinol, amines, and alcohols may also be produced. Acid and gas are produced from pyruvate and glucose, but with pyruvate as the substrate, the $p \mathrm{H}$ of the medium is not necessarily lowered because of the occasional production of acids weaker than pyruvate.

Isolated from the normal and pathological female genital tract and blood in puerperal fever, from respiratory and intestinal tracts in normal humans and animals, from the oral cavity, from pyogenic infections, putrefactive war wounds, and appendicitis. May be pathogenic.

Type species: $P$. anaerobius (Krönig) Kluyver and van Niel.

Further comment. In the author's opinion, this expanded, up-dated description is consistent with the limited original description of Peptostreptococcus (1) but is more accurate and covers a more homogeneous taxon than any previous description of which he is aware. The above description covers the type species, $P$. anaerobius, as well as the generally similar organisms $P$. foetidus (Veillon) Smith, $P$. putridus (Schottmüller) Smith, and P. lanceolatus (Prévot) Smith (4). It is reemphasized that these are not lactic acid bacteria. A second group previously included in Peptostreptococcus (Bergey's Manual, 7th ed.) are here excluded according to the present genus description; these organisms are $P$. micros (Prévot) Smith. $P$. parvulus (Weinberg et al.) Smith, $P$. intermedius (Prévot) Smith, and P. evolutus (Prévot) Smith. These organisms do not produce fetid odors, do not produce gas in culture of from pyruvate, do not produce $C_{4}$ to $C_{6}$ volatile acids, but rather produce lactic acid as a major product. These lactic acid bacteria also have the other general characteristics of Streptococcus and are simply more anaerobic or less aerotolerant than most members of the genus Streptococcus. Indeed, many strains of the presently discussed taxon, 
particularly those often designated as $P$. intermedius and $P$. evolutus, grow well in air after one or more passages.

A third heterogeneous group, previously described (4), also included in Peptostreptococcus is not herein included because the members of this group are not covered by the presently given generic description; these are $P$. magnus (Prévot) Smith, P. plagarumbelli (Prévot) Smith, $P$. paleopneumoniae (Prévot) Smith, and $P$. morbillorum (Prévot) Smith. The first two appear to have morphological characteristics consistent with those of Peptococcus, and the latter two organisms are lactic acid bacteria having characteristics consistent with those of organisms in the genus Streptococcus.

Ruminococcus Sijpestein, 1948.

Spherical to somewhat elongated cocoid cells with a diameter from 0.7 to $1.2 \mu \mathrm{m}$. When elongated, the cells tend to have rounded or flattened ends rather than pointed. When in the diplococcal arrangement, the sides of the cells may be flattened, thus resembling neisseriae and pediococci. Occur as single cells, diplococci, or short chains (usually 3 to 4 cells) or as chains of 8 to 50 or more cells. In young culture, the cells contain an iodophilic reserve polysaccharide (glucose polymer) which may rapidly disappear in the stationary phase of growth. Endospores and motility are absent. Gram-positive but may stain ambiguously. Anaerobic. Chemoorganotrophic. Complex nutritional requirements for growth are satisfied by rumen fluid or fecal extracts in usual media. The majority of strains grow in a defined medium containing $\mathrm{B}$ vitamins, cellobiose, minerals, ammonia, sulfide, $\mathrm{CO}_{2}$, acetate, and one or more of the branch-chained acids: isovalerate, isobutyrate, and 2-methyl-butyrate. $\mathrm{Am}$ monia is essential as the main nitrogen source. Amino acids are generally poorly utilized for growth, although methionine and vitamin $B_{12}$ are sometimes required. Catalase, indole, and $\mathrm{H}_{2} \mathrm{~S}$ are not produced. Starch is not degraded, nitrate is not reduced, ammonia is not produced from amino acids or peptides, the Voges-Proskauer reaction is variable, and a small minority of strains liquefy gelatin. Cellu- lose is digested (a very few noncellulolytic strains have been isolated). Cellobiose is fermented, and about $85 \%$ of the strains ferment xylan. A given strain may attack no other sugars or may attack one or more of the following: glucose, D-xylose, esculin, fructose, lactose, and L-arabinose. No acid is produced from a wide variety of other carbohydrates and polyols. In media containing bicarbonate and gassed with $\mathrm{CO}_{2}$, major products from cellobiose are acetic and formic acids; ethanol and/or succinic acid may also be major products; however, when ethanol is a major product, there is generally more hydrogen and little or no succinic acid, and when succinate is a major product there is little hydrogen and little or no ethanol. Lactic acid, if produced, is generally a minor product; methane and fatty acids containing three or more carbon atoms are not formed. Isolated from bovine and ovine rumens and from ceca of rabbits and guinea pigs. Probably widely distributed in the rumens of Ruminantia and in the cecum and colon of herbivores, and thought to play an important role in the fermentation of cellulose in these organs. The guanine plus cytosine of the deoxyribonucleic acid ranges from 39.8 to 45.4 moles per cent $\left(T_{m}\right)$.

Type species: $R$. flavefaciens Sijpestein.

\section{LITERATURE CITED}

1. Kluyver, A. J., and C. B. van Niel. 1936. Prospects for a natural system of classification of bacteria. Zentralbl. Bakteriol. Parisitenk. Infektionskr. Hyg. Abt. II 94:369-403.

2. Prévot, A.-R., and V. Fredette. 1966. Manual for the classification and determination of the anaerobic bacteria. Lea and Febiger, Philadelphia.

3. Rogosa, M. 1971. Transfer of Veillonella Prévot and Acidaminococcus Rogosa from Neisseriaceae to Veillonellaceae fam. nov. and the inclusion of Megasphaera Rogosa in Veillonellaceae. Int. J. Syst. Bacteriol. 21:231-233.

4. Smith, L. DS. 1957. Genus V. Peptostreptococcus Kluyver and van Niel, 1936, p. 533-544. In R. S. Breed, R. G. D. Murray, and N. R. Smith (ed.), Bergey's manual of determinative bacteriology, 7 th ed. The Williams \& Wilkins Co., Baltimore. 